\title{
APLICACIÓN DE LA TELEDETECCIÓN AL ESTUDIO DE LA DIFUSIÓN DE SÓLIDOS EN SUSPENSIÓN EN LOS SUCESIVOS VACIADOS DE BARASONA
}

\author{
R. Peña Martínez, A. Ruiz-Verdú, Mª.L. Serrano Pérez \\ Centro de Estudios y Experimentación de Obras Públicas (CEDEX). Paseo. Bajo de la Virgen del Puerto, 3. \\ 28005-Madrid.
}

\section{RESUMEN}

El Centro de Estudios Hidrográficos del CEDEX ha venido desarrollando trabajos de aplicación de la teledetección aeroportada a estudios de calidad del agua en ríos españoles. cuya anchura no periiiitc su estudio a través de sensores de satélite, de nienor resolución. Se ha estudiado el efecto de los vertidos térmicos sobre el río Tajo y la difusión de sólidos en suspensión en los ríos Ésera, Cinca y Segre, que es objeto del presente trabajo. Se han seguido los vaciados de los años 1995, 1996 y 1997 utilizando el sensor multiespectral ATM Daedalui 1268, del INTA, seleccionando una resolución espacial de 3.44 in en el nadir. Los mapas temáticos de sólidos en suspensión permiten observar la extensión de las zonas afectadas y la concentración en sólidos, que ha sido mucho inenor en 1996 y 1997 que en el primer vaciado en 1995.

Palabras clave: Teledetección aeroportada, calidad de aguas, sólidos en suspensión, Impacto ambiental

\section{ABSTRACT}

In the last four years, the "Centrode Estudios Hidrograficos del CEDEX" is developing airborne remote sensing methods applied to water quality studies in Spanish rivers. Such approach allows the study of local areas where the stream width is too narrow for the use of satellite imagery with larger resolution than necessary: Initially, the main application of this methodology has been the study of the thermal pollution produced by emissions of cooling water from nuclear and thermal power plants in the Tajo river basin. In this study it is followed the diffusion of suspended solid ir7 rivers Ésera, Cinca and Segre because of the withdrawal of the Barasona reservoir in 1995, 1996 and 1997. In this case we use u multispectral sensor Daedalus ATM (Airborne Thematic Mapper) of INTA with a spatial resolution of $3.44 \mathrm{~m}$ at nadir. The $\mathbf{h}$ matic maps of suspended solids shows the extension of the affected areas along the rivers and the concentration levels ut euch stretch. The obtained results show thut the impac1 of the first operation in 1995 was much more important than such of 1996 and I997.

Key words: Airborne remote sensing, Water quality, Suspended solids. Environmental impacts

\section{INTRODUCCIÓN}

\section{La teledetección: una herramienta auxiliar}

El CEDEX, a través de su Centro de Estudios Hidrográficos, lleva a cabo estudios de calidad del agua en embalses y, en general, todo tipo de aguas continentales incluso subterráneas, en todo el ámbito territorial de competencias de la Administración Central en materia de gestión de los recursos hídricos. Particularmente los estudios de calidad de agua de embalses, en los que se evalúa el estado trófico de estas masas de agua, se realizan mediante metodologías convencionales y también, desde 1985, utilizando la teledetección (Ortiz y Peña, 1989; Peña y Serrano, 1992; Peña y Serrano, 1994).
El término teledetección (remote sensing, télédétection. etc.) hace referencia a un conjunto de técnicas y métodos cuyo objetivo es la captación de información sobre un determinado objeto o fenómeno sin mantener contacto directo con él. En este ámbito se incluyen muy variados sistemas de medida u observación instalados sobre muy diversos elementos portadores (satélites artificiales, aviones, helicópteros, etc.). En un amplio sentido cabría en este marco tanto la fotografía aérea como la fotointerpretación o la fotogrametría, pero en realidad ya ocupan campos específicos hace muchos años. Por ello habitualmente la palabra teledetección se aplica a la toma de información y de forma preferente imágenes digitales, mediante sensores $o$ radiómetros situa- 
dos a bordo de satélites, aviones u otras plataformas portadoras.

Las imágenes digitales son en realidad ficheros de datos que contienen ordenadamente la cuantificación de la radiación que el sensor recibe de cada porción del terreno que abarca, que varía según la resolución del mismo. Dicha resolución es el tamaño del pixel de imagen (por ejemplo, 1 - $10 \mathrm{~m}$ en aviones, $30 \mathrm{~m}$ en el satélite Landsat, o $5000 \mathrm{~m}$ en el cotidiano Meteosat de información meteorológica, etc.). Los radiómetros situados en las plataformas portadoras reciben información analógica que transforman en digital y transmiten a estaciones terrenas. Esta técnica de teledetección. o utilización de sensores remotos, se inició hace muchos años con fines militares, pero progresivamente se va utilizando más para todo tipo de aplicaciones científicas, técnicas y de interés económico. Particularmente son importantes en este campo los llamados satélites de observación de la Tierra, como el Landsat norteamericano, el Spot francés, etc., que llevan equipos de captación de datos, o sensores, adecuados a las características de los componentes de la superficie de la Tierra.

Normalmente los sensores llevan radiómetros de varios rangos de longitud de onda o ventanas espectrales (azul, verde, rojo, infrarrojo, microondas, etc.), que están diseñados para recibir en cada unidad de información, o píxel, la radiación reflejada o emitida por una porción de la superficie terrestre más o menos amplia en función de su resolución espacial, en el caso de los satélites Landsat $30 \times 30 \mathrm{~m}$, de los Spot $20 \times 20 \mathrm{~m}$ y en sensores aeroportados entre $1.72 \times 1.72 \mathrm{~m} \mathrm{y}$ $6.88 \times 6.88 \mathrm{~m}$. De esta forma se genera una imagen digital (o banda) por cada radiómetro. Esto es lo que permite, aprovechando que cada objeto, o punto de la Tierra, produce valores distintos de radiación en las distintas bandas que constituyen su firma espectral, relacionar éstos con los datos sobre determinados parámetros y los cambios que se producen en ellos.

\section{Antecedentes}

Hasta hace algunos años los estudios de teledetección aplicada a la calidad de las aguas en el CEDEX se habían llevado a cabo haciendo uso de imágenes del sensor TM de Landsat, con 7 bandas, con información procedente de otros tantos sensores radiométricos cuya resolución espa- cial resulta adecuada para el estudio de los embalses, con alcance regional o de una cuenca hidrográfica.

Cuando se plantearon estudios cuyo ámbito de aplicación eran ríos, o masas de agua muy pequeñas y localizadas, se consideró que las imágenes obtenidas por sensores a bordo de aviones constituirían la mejor herramienta a utilizar, debido a que proporcionan mayor resolución espacial con el mismo esquema radiométrico. Existen numerosos trabajos publicados que utilizan sensores aeroportados para la estimación de parámetros de calidad de aguas (Grenier et al., 1991; Hamilton et al., 1992; Melack y Gastil, 1992; Jupp et al., 1992; Dekker y Donze, 1994; Dierber y Carriker, 1994).

Con este objetivo el CEDEX y el INTA establecieron y mantienen un convenio de colaboración para la obtención y tratamiento de imágenes digitales del sensor ATM orientadas a estudios de calidad hídrica mediante técnicas de teledetección aeroportada. Una recopilación de los sistemas aéreos y espaciales utilizados en este campo se presenta en Kirk (1983) y de teledetección aeroportada en Hilton (1984) y Danson y Plummer (1995).

Para el estudio de vertidos térmicos en el Tajo se llevaron a cabo campañas a lo largo de los veranos de 1994, 1995 y 1996. Las zonas afectadas por el estudio son las de vertido de las centrales nucleares de Trillo (Guadalajara), José Cabrera en Zorita de los Canes (Guadalajara) (1995 y 1996) y Almaraz (Cáceres) (1994 y 1995), así como de la central térmica de Aceca (Toledo) (1994 y 1995). El objetivo es la estimación del incremento de la temperatura en el río Tajo a consecuencia del vertido de agua a temperatura más alta procedente de los circuitos de refrigeración de dichas centrales. Así mismo se incluyeron otros parámetros como concentración de clorofila a y sólidos en suspensión.

En el presente trabajo se expone la aplicación llevada a cabo para la evaluación de la difusión de sólidos en los ríos Ésera, Cinca y Segre, aguas abajo del embalse de Barasona (Joaquín Costa) como consecuencia de los vaciados producidos.

Para el estudio de la difusión de sólidos en la zona previsiblemente afectada se efectuaron campañas en los otoños de 1995, 1996 y 1997, programándose los vuelos en cada caso, antes y después de los vaciados previstos. Los paráme- 
tros estimados fueron los sólidos en suspensión y la temperatura.

\section{MATERIALY MÉTODOS}

\section{Descripción de las campañas de vuelo}

Para las campañas de estudio se definieron inicialmente cuatro zonas de vuelo (zonas 1-4) que corresponden a cuatro tramos fluviales comprendidos entre el Embalse de Barasona y la confluencia del río Cinca con el Segre. Además, posteriormente, en noviembre de 1997, se definieron otras tres zonas (zonas 5, 6 y 7) en el embalse de Ribarroja al evidenciarse el interés de disponer de imágenes de esas zonas para evaluar el alcance de los efectos del vertido de sedimentos. Las campañas fueron realizadas en septiembre, octubre y noviembre de 1995, septiembre y octubre de 1996 y septiembre y octubre de 1997.

\section{Zonas de estudio}

El desarrollo lineal de los cauces presuntamente afectados superaba los $100 \mathrm{~km}$, lo que representaba una desproporcionada cantidad de información que generaría problemas de proceso de las imágenes. Para ello se definieron áreas de trabajo de dimensiones adecuadas y manejables, ya en el primer proyecto se seleccionaron, mediante reconocimiento directo del terreno, 7 pasadas, aunque no todas ellas fueron siempre cubiertas por los vuelos. Las zonas se presentan en el esquema de situación de la figura 1 del anexo.

- Zona 1 (Fraga): Comprende la confiuencia de los ríos Cinca y Segre y los últimos kilómetros del cauce del Cinca, incluyendo la ciudad de Fraga.

- Zona 2 (Ballobar): Situada aguas arriba de la anterior. comprende el cauce del río Cinca entre Chalamera y Albalate y el tramo final del río Alcanadre. No se estudió en las Últimas fechas.

- Zona 3 (Monzón): Comprende el cauce del río Cinca entre las ruinas del puente de Torre Paisanto y la Estación del SAICA aguas abajo de la ciudad de Monzón.

- Zona 4 (Barasona): Incluye la zona inmediata a la presa del embalse de Barasona, y el cauce del río Ésera desde la presa hasta su afluencia en el río Cinca, en la zona del Caladero, Puente de las Pilas, Presa de El Ciego, etc.
- Zona 5 (Segre): Se definió para cubrir el Segre hasta Mequinenza. No se ha utilizado.

-Zona 6 (Mequinenza): Comprende la zona de la confluencia del Segre con el Ebro, dentro del embalse de Ribarroja, con el pueblo y la presa de Mequinenza.

- Zona 7 (Ribarroja): Comprende la zona de la presa de Ribarroja, como control lejano del posible alcance de la influencia del vaciado.

\section{Fechas de los vuelos}

En todos los casos se siguió el criterio de realizar una toma anterior, lo más cercana posible en el tiempo, a la fecha prevista para el vaciado y otra después de transcurridos unos días, en los que se habría extendido el efecto de la circulación de sólidos por los cauces aguas abajo de la presa, para evaluar la zona afectada por la circulación de sólidos. Las fechas de cada vuelo fueron las siguientes:

$\begin{array}{lll}1995 & \text { Previo al vaciado: } & 27 \text { de Setiembre. } \\ & \begin{array}{l}\text { Posterior al vaciado: } \\ \text { Vuelo posterior: }\end{array} & 16 \text { de Octubre. } \\ & & 28 \text { de Novieinbre } \\ 1996 & \begin{array}{l}\text { Previo al vaciado: } \\ \text { Posterior al vaciado: }\end{array} & \begin{array}{l}25 \text { de Setiembre } \\ 10 \text { de Octubre. }\end{array} \\ & & \\ 1997 & \text { Previo al vaciado: } & 30 \text { de Setiembre } \\ & \text { Posterior al vaciado: } & 8 \text { de Octubre. }\end{array}$

Tabla 1. Configuraciones posibles del sensor ATM. Available configurations of the ATM sensors.

\begin{tabular}{clll}
\hline CANAL & ATM DS-I 26X & DS-1268 op.3-5 & CZCS \\
\hline 1 & $0.42-0.45 \mu \mathrm{m}$ & $0.42-0.45 \mu \mathrm{m}$ & $0.423-0.463 \mu \mathrm{m}$ \\
2 & $0.45-0.52 \mathrm{TM} 1$ & $0.45-0.52 *$ & $0.48-0.50$ \\
3 & $0.52-0.60 \mathrm{TM} 2$ & $0.52-0.60 *$ & $0.508-0.532$ \\
4 & $0.60-0.62$ & $0.60-0.62$ & $0.543-0.577$ \\
5 & $0.63-0.69 \mathrm{TM} 3$ & $0.63-0.69 *$ & $0.58-0.63$ \\
6 & $0.69-0.75$ & $0.69-0.75$ & $0.638-0.70$ \\
7 & $0.76-0.90 \mathrm{TM} 4$ & $0.76-0.90 *$ & $0.715-0.815$ \\
8 & $0.91-1.05$ & $0.91-1.05$ & $0.83-0.94$ \\
9 & $\mathrm{I.55}-1.75 \mathrm{TM} 5$ & $1.55-1.75 *$ & $1.55-1.75$ \\
10 & $2.08-2.35 \mathrm{TM} 7$ & $2.08-2.35$ & $2.08-2.35$ \\
11 & $8.5-13.0 \mathrm{TM} 6$ & $3.0-5.0 *$ & $8.5-13.0$ \\
12 & $8.5-13.0$ & $8.5-13.0 *$ & $8.5-13.0$ \\
\hline
\end{tabular}

ATM = Airhornc Thematic Mapper CZCS = Coastal Zone Colou Scanner 


\section{Sensor utilizado}

Se ha empleado un sensor de barrido multiespectral (que recoge información de varias ventanas del espectro y toma la imagen mediante un dispositivo que va desplazando transversalmente el haz del sensor conforme avanza el vehículo). Es el modelo DAEDALUS 1268 que es de tipo pasivo, es decir recibe radiación emitida o reflejada, y va instalado en un avión CASA-212 modificado, que separa la radiación recibida de la superficie terrestre en once canales espectrales desde el azul hasta el infrarrojo térmico, dependiendo de la configuración espectral seleccionada. Además permite obtener imágenes de distinta resolución espacial. Para los presentes trabajos se ha escogido un tamaño de pixel de $3.44 \mathrm{~m}$ en el nadir.

El sensor es de estructura modular, programable según las necesidades de cada misión o aplicación. Las configuraciones que puede adoptar el sensor se recogen en la Tabla I. En ella figuran marcadas con un asterisco $\left({ }^{*}\right)$ las bandas que se han seleccionado para este estudio.

El sensor se ajusta y se calibra en un banco especialmente diseñado para esta tarea situado en el laboratorio de teledetección del INTA. Antes de su utilización para la obtención de mapas temáticos, las imágenes son deconmutadas mediante un sistema de conversión específico que, a partir de las cintas de alta densidad grabadas en el mismo avión, estructura los datos en formato ELAS sobre cintas magnéticas compatibles. Posteriormente, debido a que su baja altura de vuelo produce distorsiones sistemáticas progresivas desde el eje a los extremos de la pasada, las imágenes se someten a una corrección panorámica utilizando un algoritmo desarrollado en el INTA de acuerdo con las consideraciones geométricas definidas por el propio sistema de adquisición. Por Último se graban en CD-ROM para su manipulación y proceso posteriores.

\section{Proceso de datos}

Para la modelización de los sólidos en suspensión se lleva a cabo un análisis estadístico de datos y un proceso de ajuste, consistente en regresiones lineales múltiples mediante un procedimiento de iteración selectiva (Verdin, 1984). Como variables independientes figuran los valores de ND registrados en los puntos de muestreo localizados en los canales espectrales, mientras que la variable dependiente es la concentración de sólidos en suspensión medida en cada uno de dichos puntos. Los canales utilizados para las ecuaciones son el 2, 3, 5,7 y 9 del sensor ATM, que emulan, es decir reproducen la tnisma ventana espectral, a las bandas 1, 2, 3,4 y 5 del sensor TM de Landsat respectivamente.

Para la conversión de los niveles digitales que proporciona el sensor en las bandas térmicas $11 \mathbf{y}$ 12 a valores de temperatura, se utilizan los dos cuerpos negros (emisividad $>0,99$ ) de referencia, frío y caliente, para los cuales se dispone de pares Temperatura - ND registrados para cada línea de imagen. Estos pares se promedian y los dos puntos así obtenidos definen una recta que permite obtener el valor de temperatura (T) para cada nivel digital (ND):

$$
\mathrm{T}=\mathrm{ND} * \mathrm{~m}+\mathrm{n}
$$

Hay que tener presente que $T$ es la temperatura aparente del terreno, sin considerar la interferencia atmosférica ni la emisividad de la superficie. Para todas las campañas, se ha obtenido que $\mathrm{m}=0.18$, representando la resolución térmica de las imágenes en ausencia de ruido instrumental.

La elaboración de mapas temáticos de sólidos en suspensión y clorofila consiste en la aplicación de las regresiones múltiples obtenidas a partir de los canales del sensor. En el caso de la temperatura se aplica la ecuación (1) al canal 12 (infrarrojo térmico), corregida en cada imagen con los datos de campo. Las imágenes de estos canales se cargan en un Sistema de Información Geográfica con modulo de tratamiento de imágenes en el que cada canal opera como una capa de información obteniéndose "mapas resultado" de sólidos en suspensión o temperatura en función de la ecuación aplicada, ya que dichos factores se aplican a todos y cada uno de los puntos de las bandas relacionadas en la ecuación. La metodología se describe en trabajos anteriores (Serrano y Peña, 1997).

\section{RESULTADOS}

Se han obtenido ecuaciones de concentración de sólidos en suspensión para cada una de las fechas de estudio. Estas ecuaciones y sus coeficientes de determinación $\left(\mathrm{r}^{2}\right)$ se resumen en la Tabla 2. 
Tabla 2. Ecuaciones representativas de la concentración de sólidos en suspensión. Adjusted equations for the calculation of the suspended solids concentration.

\begin{tabular}{llll}
\hline Fecha & Zola & Ecuación & $\mathrm{r}^{2}$ \\
\hline $27 / 09 / 95$ & $1,2,3,4$ & {$[\mathrm{ss}]=72.05-2.16 * \mathrm{~B} 2+2.57 * \mathrm{~B} 50.82 * \mathrm{~B} 9$} & 0.67 \\
$16110 / 95$ & $1,2.3,4$ & {$[\mathrm{ss}]=371.48 * \mathrm{~B} 3-1218.66$} & 0.76 \\
$28111 / 95$ & 1.3 .4 & {$[\mathrm{ss}]=418.01 * \mathrm{~B} 2-15658.60$} & 0.87 \\
$28 / 11 / 95$ & 6 & {$[\mathrm{ss}]=7.84 * \mathrm{~B} 2-324.48$} & 0.97 \\
$28 / 11 / 95$ & 7 & {$[\mathrm{ss}]=0.51 * \mathrm{~B} 3-0.24 * \mathrm{~B} 2-0.06$} & 0.99 \\
25109196 & 1.3 .4 & {$[\mathrm{ss}]=4.14 * \mathrm{~B} 2-96.04$} & 0.89 \\
$25 / 09 / 96$ & 6 & {$[\mathrm{ss}]=0.60 * \mathrm{~B} 5-11.53$} & 0.83 \\
$10 / 10196$ & 1.3 .4 & {$[\mathrm{ss}]=80.67 * \mathrm{~B} 3-2.57 .09$} & 0.92 \\
$10 / 10196$ & 6 & {$[\mathrm{ss}]=0.9203 * \mathrm{~B} 5-15.92$} & 0.83 \\
30109197 & $1,3.5,6$ & $\log (\mathrm{ss})=0.0038 * \mathrm{~B} 2+0.034 * \mathrm{~B} 7-0.438$ & 0.82 \\
30107197 & 4 & {$[\mathrm{ss}]=0.248 * \mathrm{~B} 3-6.758$} & 0.71 \\
$08 / 10 / 97$ & $1,3,4,5,6 \ln (\mathrm{ss})=0.045 * \mathrm{~B} 3+0.108 * \mathrm{~B} 7+0.032$ & 0.95 \\
\hline
\end{tabular}

Las ecuaciones sirven de base para la elaboración de los mapas temáticos. De la observación de la cartografía temática se obtiene un esquema de distribución de sólidos que se resume en la Tabla 3 así como en los gráficos de barras de la figura 2 del anexo.

Se puede observar en los mapas resultantes que el primer vaciado del embalse, realizado en el otoño de 1995, produjo un gran aumento de la concentración de sólidos en suspensión en el río Cinca, que se mantiene en el tramo del río Segre desde que recibe al Cinca hasta su unión con el Ebro en el embalse de Ribarroja.

De una concentración de sólidos, que variaba entre 20 y $100 \mathrm{mg} / \mathrm{l}$ en las pasadas previas, se pasó a una concentración entre 5 y 10 g/l, e incluso superior en algunos tramos.
En el año 1996 los incrementos fueron algo menores, alcanzándose valores entre I y $4 \mathrm{~g} /$ l. Los mapas obtenidos muestran que estos valores se mantienen a lo largo del río Cinca hasta después de la confluencia con el río Segre. Después, la concentración de sólidos en suspensión disminuye progresivamente conforme el agua del Cinca se va adentrando en el embalse de Ribarroja, hasta alcanzar valores de unos 100 $\mathrm{mg} / \mathrm{l}$. Esta Ultima zona es, posiblemente, el lugar donde sedimentan la mayor parte de los sólidos procedentes del embalse de Joaquín Costa.

En la operación realizada el año 1997 se generó una cantidad inferior de lodos y por ello el efecto también fue más reducido, aunque con algunas excepciones locales, a lo largo de los cauces dominados por el embalse.

En las figuras 3, 4 y 5 del anexo se incluyen algunos ejemplos de mapas temáticos del estudio.

\section{Evolución de la superficie de agua en el azud de El Ciego}

Es evidente que uno de los efectos inmediatos de la circulación masiva de aguas con elevadas concentraciones de sólidos en suspensión es la sedimentación de éstos a lo largo de las zonas por las que discurren, en función de su granulometría y de las condiciones del cauce que condicionan la velocidad de la corriente $y$, en función de la misma, la posibilidad de que vaya produciendo la deposición de materiales más o menos gruesos.

Ello determina que en algunos puntos, especialmente donde hay retención apreciable, se produzca acumulación de sedimentos en mayor proporción y así se pueda modificar el equilibrio existente entre las proporciones de agua y tierra

Tabla 3. Reaunien de rangos de sólidos en suspensión (concentración eii mg/l) obtenidos de los inapas temáticos. Ranges of suspended solids concentrations (in $\mathrm{mg} / \mathrm{l}$ ) obtained from the thematic maps.

\begin{tabular}{|c|c|c|c|c|c|c|c|}
\hline ZONA / FECHA & 27.09 .95 & 16.10 .95 & $28.1 \mathrm{I} .95$ & 25.09 .96 & 10.10 .96 & 30.09 .97 & 08.10 .97 \\
\hline Embalse Barasona & 3.7 & & & 133.7 & & 4.2 & \\
\hline Azud El Ciego & 10.8 & 8242.4 & 6366 & 106.2 & 2463.5 & I.6 & 1203.7 \\
\hline Zona de Monzón & 17.5 & 7893.2 & 4036.4 & 98.5 & 2448.7 & 19.6 & 2728.4 \\
\hline Fraga - Río Cinca & 49 & 6936.4 & 8816 & 61 & 2335.5 & 76.2 & 1856.8 \\
\hline Fraga - Río Segre & 92.7 & 30.2 & 208.4 & 19.6 & 115.1 & 12.5 & 36 \\
\hline Ribarroja - Segre & & & 136.5 & 28.8 & 43.7 & 25 & 63.8 \\
\hline Ribarroja - Ebro & & & 24.2 & 7.8 & 11.6 & & 3.2 \\
\hline Unión Ebro-Segre & & & 30.3 & 16.5 & 24.I & & 8.8 \\
\hline
\end{tabular}


en esas zonas. Tal es el caso del Azud de Arias o de El Ciego. En esta zona embalsada se ha producido una regresión de la lámina de agua en beneficio del área ocupada por sedimentos que, como se aprecia en el paisaje del lugar de forma muy aparente, ha permitido la ocupación de estos nuevos terrenos con abundante vegetación típica de estas zonas húmedas.

\section{CONCLUSIONES}

El planteamiento del Proyecto, dentro del marco general de estudios científicos y técnicos llevados a cabo con motivo de los vaciados, el desarrollo de los trabajos y finalmente los resultados obtenidos, con la cartografía temática de las zonas de estudio, ponen de manifiesto la viabilidad de acometer este tipo de trabajos con una herramienta como las imágenes digitales adquiridas por sensores radiométricos abordo de aviones.

La teledetección con este tipo de información radiométrica utiliza todas las posibilidades de modelización de parámetros que suministran los sensores de los satélites, como Landsat o Spot, pero incorporando una resolución espacial que permite discriminar fenómenos más concretos, con más detalle, como en el caso de corrientes de agua superficiales.

La teledetección aeroportada es una herramienta muy flexible en cuanto a periodicidad y elección de fechas ya que facilita la posibilidad de planificación de los vuelos. Ello es muy útil en aquellas áreas en las que la frecuente cobertura nubosa exige gran capacidad para adaptarse a las condiciones meteorológicas a la hora de adquirir las imágenes, que en muchas ocasiones resulta imposible conseguir.

Por otra parte, en lo que respecta a la difusión de los sedimentos a lo largo de los cauces aguas abajo del embalse, a través de la cartografía temática producida se consigue una clara visión de la gran concentración de sólidos en suspensión que se alcanzó en octubre-noviembre de 1995 , llegando el efecto con apreciable intensidad hasta por lo menos la unión del Ebro y el Segre en el embalse de Ribarroja, más allá de la población de Mequinenza, que luego va desapareciendo, diluida progresivamente en el embalse, que además tiene un tiempo de retención del agua muy corto, y por ello gran tasa de circulación a través de su vaso. En cambio, en el año 1996 el nivel de concentración que se alcanzó fue muy inferior, aunque el efecto se apreciara también, pero sin apenas importancia en la confluencia citada. Por último, en 1997 el impacto es aún menor en todas las zonas, ya que, como es lógico, el volumen de materiales depositados en el fondo del vaso de Barasona eran cada vez menores debido a los sucesivos vaciados, tan poco separados en el tiempo.

Finalmente hay que destacar que la herramienta utilizada en este trabajo ha permitido valorar los efectos del vertido de sólidos en toda su extensión, lo que no es posible abarcar en otros estudios realizados con este motivo que, por razones obvias, han tenido que referirse a las zonas más cercanas alrededor del embalse de Joaquín Costa y los cauces más inmediatos.

\section{BIBLIOGRAFÍA}

DANSON F.M. \& S.E. PLUMMER. 199.5. The Remote Sensing of Inland Water Quality.Advances in Environmental Remote Sensing. 8:123-142.

DEKKER, A. G. \& M. DONZE. 1994. Imaging Spectrometry as a research tool for inland water resources analysis. In: Imaging Spectrometry - $\boldsymbol{A}$ tool for environmental applications. J. Hill (ed.): 295-3 18. Kluwer Academic. Dordrecht.

DIERBERG, F.E. \& N.E. CARRIKER. 1994. Field testing two instruments for remotely sensing water quality in the Tennessee Valley. Environmentul Science and Technology, 28:16-25.

GREENER, M. 1991. Evaluation de la qualité des eaux du fleuve Saint-Laurent par téledétection aeroportée. Montreal: Centre Saint-Laurent de Conservation et Protection de l'Environement, Canada.

HAMILTON, M. 1992. Multiple dataset water-quality analysis in the vicinity of an ocean wastewater plume. Proceedings of the $3^{\text {rd }}$ Airborne Geosciences Workshop. Pasadena. JPL92, 14:63-65.

HILTON. J. 1984. Airborne remote sensing for freshwater and estuarine monitoring. Water Resources, I8:1195-I 223.

JUPP, D.L.B. \& J. PARSLOW. 1992. The potential use of airborne scanning for monitoring algal blooms in the Murray Darling Basin, Report to the Murray Darling Basin Commission: Airborne 
Remote Sensing of blue-green algae at Lake Mokoan. Canberra: CSIRO Division of Water Resources and CSIRO-OSSA.

KIRK, J.T.O. 1983. Light and photosynthesis in Aquatic Ecosysterns. Cambridge University Press. Cambridge.

MELACK, J.M. \& M. GASTIL. 1992. Seasonal and spatial variations in phytoplanktonic chlorophyll in eutrophic Mono Lake, California, measured with the AVIRIS. Proceedings of the $3^{\text {rd }}$ Airhorne Geosciences Workshop. Pasadena. JPL92, 14:5355.

ORTIZ, J.L., R. PEÑA. 1989. Remote Sensing of Limnological Parameters in reservoirs. En: Proceedings of a Workshop on Earthnet Pilot Project on Landsat Thematic Mapper Applications. Frascati. ESA SP-1 102: 49-56.

PEÑA, R., A. FERNÁNDEZ-RENAU, E. DE MIGUEL, S.A. GÓMEZ, M.L. SERRANO y A. RUIZ-VERDÚ. 1997. Utilización de sensores aeroportados en aplicaciones a la calidad del agua: eva- luación de vertidos térmicos en el río Tajo y difusión de sedimentos en los ríos Esera y Cinca. En:Teledetección aplicado a la gestión de recursos naturales y medio litoral marino. Carlos Hernández Sande, Juan E. Arias Rodríguez (eds.): 335-339. Pub. Asociación Española de Teledetección. Universidad de Santiago de Compostela.

PEÑA, R. y M.L. SERRANO. 1992. Evaluación del estado trófico de los embalses mediante imágenes digitales. Ingeniería Civi1, 86:37-44.

PEÑA, R. y M.L. SERRANO. 1994. Seguimiento del estado trófico de los embalses del alto Tajo por teledetección. Limnética, 10:69-81.

SERRANO, M.L. y R. PEÑA. 1997. Evolución del estado trófico de tres embalses del Júcar por teledetección. En: Teledetección. Usos y aplicaciones. J.L. Casanova y J. Sanz (eds.): 223-229. Pub. Universidad de Valladolid.

VERDIN, J.P. 1984. Limnological applications of Multispectral remote sensing. USBR Report No.REC-ERC-85-2. $51 \mathrm{p}$ 